\title{
Des vergers de Pandanus spp. comme poste avancé de la culture
}

Notule d'ethnobotanique baruya

Pierre Lemonnier

\section{(2) OpenEdition}

\section{Journals}

Édition électronique

URL : http://journals.openedition.org/jso/1480

DOI : $10.4000 /$ jso. 1480

ISSN : $1760-7256$

\section{Éditeur}

Société des océanistes

Édition imprimée

Date de publication : 1 décembre 2002

Pagination : 159-164

ISSN : 0300-953x

Référence électronique

Pierre Lemonnier, «Des vergers de Pandanus spp. comme poste avancé de la culture », Journal de la Société des Océanistes [En ligne], 114-115 | Année 2002, mis en ligne le 26 mai 2008, consulté le 22 mars 2021. URL : http://journals.openedition.org/jso/1480 ; DOI : https://doi.org/10.4000/jso.1480

\section{(c) $\oplus \Theta \Theta$}

Journal de la société des océanistes est mis à disposition selon les termes de la Licence Creative Commons Attribution - Pas d'Utilisation Commerciale - Pas de Modification 4.0 International. 


\title{
Des vergers de Pandanus spp. comme poste avancé de la culture.
}

\author{
Notule d'ethnobotanique baruya
}

par

\author{
Pierre LEMONNIER*
}

\section{RÉSUMÉ}

Chaque année, vers la mi-octobre, un quart de la population baruya s'enfonce dans la forêt avec cochons, chiens et enfants, bien au-dessus des jardins les plus élevés, pour collecter, surveiller et conserver les fruits d'arbres "semi-domestiqués » de la forêt, les Pandanus jiulianettii et $\mathrm{P}$. brosimos. Installés pendant deux mois dans des petits hameaux rassemblant de solides bâtisses, hommes et femmes font sécher par centaines de kilogrammes des drupes ou des morceaux de syncarpes de Pandanus au-dessus de feux qui brûlent jour et nuit. Le gros de la consommation des (délicieuses) amandes intervient pendant la mauvaise saison (froide et très pluvieuse). Avec $66 \mathrm{~g}$ de graisse pour $100 \mathrm{~g}$ de matière sèche, les amandes sont, de loin, le plus riche des aliments que consomment les Baruya. C'est aussi celui qui contient le plus de protéines. On peut alors avancer l'hypothèse que, moins touchés par l'absence de pluie dans l'écrin humide que leur offre la forêt tropicale d'altitude, les Pandanus offrent aux Baruya une nourriture de famine d'une exceptionnelle qualité. Ces postes avancés de la culture des plantes que sont les bosquets de Pandanus sont aussi les seuls lieux de production agricole qui, pendant plusieurs mois, demandent une présence de l'homme de tous les instants. De ces arbres pas comme les autres auprès desquels leur société se morcelle par clan et lignage pour résider et travailler ensemble, les Baruya font également des théâtres végétaux lors des cérémonies d'initiation.

Mots-CLÉS: Nouvelle-Guinée, Anga, Pandanus, domestication, nutrition.

\section{ABSTRACT}

Each year, towards mid-October, a quarter of the Baruya population heads up into the forest with their pigs, dogs and children, well above the highest cultivated gardens, to collect, watch over and preserve the fruits of " semi-domesticated " forest trees, the Pandanus jiulianettii and $\mathrm{P}$. brosimos. There, in their solidly constructed hamlets, men and women spend two months drying hundreds of kilos of the Pandanus drupes or pieces of syncarpes over fires that are kept burning day and night.The bulk of the (delicious) almonds are consumed during the cold, rainy season. With $66 \mathrm{~g}$ of fat for $100 \mathrm{~g}$ of dehydrated matter, almonds are by the far the richest food consumed by the Baruya. They are also the food that is highest in protein. One can therefore emit the hypothesis that, protected from the lack of rainfall by their high rain forest, the Pandanus provides the Baruya with an exceptionally high-quality food in times of famine. These Pandanus grove out-posts are also the only sites of agricultural production which require an uninterrupted human presence for several months a year. These trees unlike any others, around which the Baruya society gathers by clans and lineages to live and work together, are also used by the Baruya as vegetal theaters in their initiation ceremonies.

Keywords : New Guinea, Anga, Pandanus, domestication, food. 
«Les fruits de ces Pandanus sont souvent conservés dans des huttes ou encore dans des greniers situés au-dessus des cases. Les autochtones entretiennent constamment des foyers dans leurs habitations, aussi les fruits de Pandanus qui y sont emmagasinés subissent-ils un fumage permanent qui facilite leur conservation » (Barrau, 1962 : 193)

« On est en train de se battre parce que, tout là-haut, quelqu'un a coupé un karuka! ». Même replacé dans le contexte de l'histoire récente de la vallée de Wonenara (Eastern Highlands, province de Papouasie Nouvelle-Guinée) — la dernière où les Baruya s'installèrent il y a un peu plus d'un siècle après avoir repoussé les Yuwarunatse vers le nord - , le motif paraissait assez maigre : quelque part dans la forêt d'altitude, dans un minuscule recoin de l'immense no man's land qui sépare les territoires baruya, yuwarunatse et watchakes, " on» (Baruya? Yuwarunatse ?) avait abattu un karuka (nom en tok pisin pour Pandanus brosimos et $P$. jiulianettii) et « on » avait trouvé l'incident suffisamment grave pour reprendre immédiatement les combats interrompus par l'arrivée des Australiens, une trentaine d'années plus tôt (1951). Il est vrai qu'avec l'Indépendance (1975), les " patrouilles" administratives et policières étaient devenues moins fréquentes. Difficile aussi de ne pas remarquer que, dans une société dont les combattants illustres sont les plus grands des grands hommes, les risques de guerre sont accrus. En tout cas, c'est ce que semblaient penser les dizaines de jeunes initiés qui, vers la même époque, s'affairaient à fabriquer arcs et boucliers devant la maison des hommes de Wiobo.

Les combats, donc, avaient repris. Le kiap (fonctionnaire chef de poste) avait plié bagages et l'administration papoue avait fermé l'école et le dispensaire de Wonenara : lorsque les Baruya et les Yuwarunatse cesseraient de combattre, l'État leur offrirait à nouveau ses services.

Pour l'heure, en novembre 1983, la guerre avait déjà fait cinq ou six morts, notamment un Yuwarunatse dont Jean-Luc Lory et moi venions d'entendre célébrer la mise hors de combat. Entonné par des centaines d'hommes perchés sur le grand rocher qui domine Yanyi, à plus de trois kilomètres de nous, le chant qui parvenait jusqu'à nous était trop faible et trop haché par le vent pour que quiconque puisse préciser qui avait porté le coup mortel ou quel ennemi avait été tué. Mais c'était une victoire. Un peu plus tard dans la soirée, nous apprîmes que le Yuwarunatse abattu était un aulatta, un grand guerrier. Et, plus précisément, celui qui n'hésitait pas à s'avancer au milieu de « nos » lignes en marchant nonchalamment, la tête baissée, son arc posé en travers de ses épaules, comme s'il était un ami. Au dernier moment, ce personnage autant haï qu'admiré relevait la tête et tirait une volée de flèches vers ses ennemis baruya avant de se mettre à couvert.

Le succès fut célébré comme il se devait, lors d'une cérémonie organisée le lendemain à la fois pour crier aux Yuwarunatse tout le mépris qu'on leur portait et pour se purifier du sang qui avait coulé. Peu après, il s'avéra que l'aulatta supposé avoir été criblé de flèches et étrangement défiguré ( « on lui a cassé le nez avec une pierre parce qu'il pensait mal ; on lui a brisé les dents parce qu'il parlait mal ») avait plutôt reçu une décharge de chevrotines en pleine tête. On nous avait pourtant affirmé que nul ne ferait usage de l'unique fusil de chasse de la vallée... Comme nous en connaissions parfaitement le propriétaire et que les hélicoptères de la police faisaient du rasemottes au-dessus de notre toit, Jean-Luc et moi décidâmes de franchir la montagne et de passer dans l'autre vallée baruya, celle de Marawaka, non sans laisser sur notre porte un écriteau indiquant fièrement que la maison était une joint property du CNRS et du Papua New Guinea Institute of Medical Research. Je crois même que nous avions ajouté « Not to be burnt! » Précision apparemment efficace car l'édifice jadis bâti par Maurice Godelier fut épargné par les grenades incendiaires qui, chaque matin, rappelaient aux Baruya (et aux Yuwarunatse) que l'État papou veillait. Partant du fait — d'ailleurs indéniable — qu' « il y a des arbres plein la forêt », et affirmant que reconstruire une hutte n'est qu'une question de temps - ce qui n'est pas faux non plus - , les uns et les autres ne se souciaient guère de cette répression, sauf pour s'assurer que la police détruisait bien un nombre égal de maisons dans chaque camp ! Pour tout dire, il régnait une atmosphère de douce rigolade, sauf les jours où « nous » avions un tué, bien entendu.

\section{Un campement forestier baruya}

Tout autant que le désir de revoir l'un de ces lieux impressionnants où les Pandanus dressent leurs couronnes de feuilles quinze ou vingt mètres au-dessus du sol, c'est l'envie de goûter ce bonheur harassant qui consiste à grimper des pentes invraisemblables dans la forêt tropicale humide qui m'amena ce jour-là à suivre Nouriandaye au-dessus de Marawaka. Une fois dépassés les champs irrigués où les Baruya cultivent les Coix gigantea dont ils tirent leur célèbre monnaie de sel (Godelier, 1969; Lemonnier, 1984), il nous fallut une petite heure pour arriver 
au plus proche de ces yashwanala (« bosquet de Pandanus à noix », Lloyd 1992 : 594) vers lesquels nous voyions chaque jour monter des femmes croulant sous des filets de portages pleins à craquer de tubercules.

Le GPS n'existait pas encore mais nous devions nous trouver vers 2300 mètres lorsque nous débouchâmes dans une fausse clairière j'entends par là un endroit au sol parfaitement dégagé et nettoyé où le regard pouvait porter à plus de cinquante mètres sans rencontrer le mur de la forêt. Là, entouré de dizaines de Pandanus «à noix », se trouvait un minuscule hameau composé, non pas de ces auvents érigés en une heure qui constituent l'habitat forestier habituel, mais de vraies maisons, trapues, bien finies et où il devait faire bon dormir au sec et au chaud.

De plan quadrangulaire, sans plancher et dotées d'un toit à deux pentes, elles n'avaient rien à voir avec les huttes en forme de ruche qui sont l'une des caractéristiques des villages anga. Solidement bâties et d'une surface au sol de 10 à 12 mètres carrés, elles étaient couvertes à l'aide de feuilles de pandanus maintenues en place par des lianes et des rondins. Leurs murs constitués de larges planches jointives plantées verticalement étaient extérieurement doublés de feuilles de pandanus horizontales. Appuyés au mur ou entassés à sa base, des dizaines de morceaux de bois de chauffe constituaient une protection supplémentaire contre le vent et le froid de la nuit (il fait entre $4^{\circ} \mathrm{C}$ et $8^{\circ} \mathrm{C}$ à l'aube). D'autres feuilles de pandanus tapissaient l'intérieur des murs, mais seulement jusqu'à une hauteur d'environ $1,20 \mathrm{~m}$. Le sol démuni de plancher était partiellement recouvert d'une accumulation de feuillages dans sa partie réservée au couchage des habitants, non loin du foyer.

Sous le toit de chaque maison, qui s'élevait jusqu'à 3 mètres de haut, des clayonnages superposés d'une surface unitaire de 2 à $2,5 \mathrm{~m}^{2}$ avaient été construits, plus ou moins à la verticale de l'unique foyer. Là étaient déposés des syncarpes de Pandanus brosimos ou de P. jiulianettii coupés en deux ou en quatre (les Anglais parlent de $n u t$ clusters pour décrire ces paquets de drupes encore accolés les uns aux autres). Une large partie du sol était recouverte d'un entassement de drupes que les femmes avaient préalablement désolidarisées. Au total, j'estime qu'il y avait environ $1,5 \mathrm{~m}^{3}$ de « noix » de pandanus en train de sécher dans chaque maison. Car c'est bien d'abord de séchage que l'on se préoccupait de la mi-octobre à la mi-décembre dans ces minuscules hameaux ouverts dans la forêt éternellement humide qui s'accroche aux pentes de la cordillère centrale de la Nouvelle-Guinée constituant le territoire baruya.

Selon les explications que je recueillais sur place, l'une des fonctions de cet habitat d'altitude où le quart (ou le cinquième ?) de la population résidait — et réside toujours chaque année — pendant deux mois est « d'être là », immédiatement sur place, au moment où le vent fait tomber au sol les syncarpes les plus mûrs, avant que les cochons sauvages et les rats ne les mangent. D'autres mirebuta (" ripe pandanus nut », Lloyd, 1992 : 594) sont coupés par les hommes qui grimpent plusieurs fois par jour le long des immenses troncs au risque de se fracasser les os sur les racines aériennes hérissées de piquants. Une fois séchées et fumées pendant deux à trois semaines au-dessus de feux qui brûlent jour et nuit, les drupes ou les morceaux de syncarpes sont descendus vers les villages de la vallée par les femmes qui, au retour, apportent les légumes à feuilles, la canne à sucre et les tubercules dont chacun s'alimente ${ }^{1}$.

\section{Une arboriculture à la marge de l'horticulture}

Comme tous les Anga, les Baruya sont des horticulteurs, c'est-à-dire des agriculteurs cultivant de petits champs ( 0,1 à 2,5 ha) qui sont le plus souvent défrichés dans la forêt. La chasse est marginale pour l'alimentation, mais le gibier (marsupiaux, porcs sauvages, casoars) tient une place fondamentale dans les rituels d'initiation et dans les échanges (entre affins notamment).

Les jardins anga ont été décrits par Lory (1982) et comparés à d'autres systèmes horticoles lors des rapides (mais denses) surveys réalisés en 1982 et 1990 dans le cadre des études qui ont abouti aux Working papers de la série « Agricultural Systems of Papua New Guinea » (Bourke et al, 1994) ${ }^{2}$. Les deux principales plantes alimentaires sont aujourd'hui la patate douce (Ipomea batatas), qui constitue environ les trois quarts de l'alimentation quotidienne, et le taro (Colocasia esculenta). Les cochons domestiques ( 0,6 par personne en 1985 , soit environ 3,3 porcs par $\left.\mathrm{km}^{2}\right)^{3}$ sont nourris (par les femmes) de patates douces crues (mais lavées).

1. Dans d'autres régions de Nouvelle-Guinée (Greve et al., $1994: 1068$ ), les drupes sont mises à fermenter dans la boue ou dans l'eau puis séchées au soleil. Rien de semblable n'existe chez les Anga.

2. On trouvera dans Lemonnier (1982) une comparaison rapide des techniques agricoles des divers groupes anga.

3. L'important n'est pas tant le nombre d'animaux par personne que le nombre d'animaux qui doivent être nourris sur un territoire donné, c'est-à-dire leur densité (Lemonnier, 1990 : 139-140 et, pour un avis contraire, Kelly, 1988). 
L'étude de Lory (1982) a montré la variété des jardins baruya selon leur altitude et leur éloignement des fonds de vallée. Dans les fonds de vallée, au pied des villages, se trouvent des champs de cannes à sel et des tarodières irriguées. Dans les villages eux-mêmes, entre 1600 et 2000 mètres d'altitude, les enclos domestiques contiennent des jardins semi-permanents (patates douces, canne à sucre, bananiers, Setaria palmifolia), dont une partie ou une autre est remise en culture tous les deux ou quatre ans. Au-dessus des villages commence la zone des jardins mixtes (patates douces et taros), souvent immenses et collectifs, dans laquelle la forêt reprend ses droits après chaque mise en culture. Plus haut encore, entre 2200 et 2400 mètres, les Baruya ouvrent dans la forêt « primaire » de très grands jardins de taros, notamment lorsqu'il s'agit d'approvisionner les festins par lesquels s'achèvent les cérémonies d'initiation. Dans ce dernier cas, la période de friche peut atteindre 25 ou 30 ans (Lory, 1982 : 251-253). La variété des types de recrus - arborés, cannes (Saccharum spontaneum, Phragmites karka, Miscanthus floridulus, etc.), herbes courtes - est une autre caractéristique de l'horticulture baruya (Bourke et al, $1994: 58)$.

Cette horticulture est loin d'atteindre l'intensité que l'on peut observer ailleurs en NouvelleGuinée, en particulier là où une grande partie des tubercules est utilisée pour nourrir les cochons. Par exemple, les Baruya n'ont pas recours au labour à bras, à la fabrication de compost, à la fumure animale ou au billonnage. Ils n'en ont pas moins des pratiques horticoles sophistiquées - ample circulation des boutures de taros (Lory, 1982: 260 et suiv.), présence de pépinières irriguées, culture du Coix gigantea. En tout état de cause, on est fort loin de cette incipient agriculture que Feil (1987 : 101) attribue à tort aux Anga.

Le territoire baruya est trop élevé pour qu'y poussent des arbres fruitiers comme la marita (nom en tok pisin pour Pandanus conoideus ${ }^{4}$ ), le Pangium edule ou les Canarium sp. Les plus importants de ces fruitiers de forêt sont sans conteste les Pandanus « à noix », Pandanus jiulianettii et $P$. brosimos, sauvages ou plantés ${ }^{5}$. Les
Baruya nomment huit cultivars de karuka (Lloyd, 1992 : 456). "Plantés », signifie ici que les hommes déterrent les syncarpes tombés au sol qui ont commencé de germer. Ils les arrangent sur le sol et nettoient les alentours de leur plantation, effectuant l'une des opérations techniques que Jacques Barrau associait à la « protodomestication » (Barrau, 1965 : 58-59). Il arrive également que les Baruya coupent une «couronne» (« infrutescence terminale et pendante ", Barrau, 1962 : 192) en haut d'un vieux Pandanus et la plantent dans le sol (Godelier, comm. pers.), ce qui est une manière d'être sûr que le nouvel arbre portera des fruits car, ainsi que l'écrit Sillitoe (1983: 107): «a seedling might be a useless male tree, whereas crowns from female trees are sure to produce balls of nuts $»$.

\section{Sociologie sommaire d'un arbre polyvalent}

Bien plus que le fumage ou la conservation des syncarpes de Pandanus, bien connus en Nouvelle-Guinée, c'est la manière dont les Baruya les organisent qui doit retenir l'attention ${ }^{6}$. Dans le campement que j'ai visité, deux des quatre maisons abritaient deux familles. À y regarder de plus près - en l'occurrence de plus haut -, les forêts baruya sont constellées de tels habitats temporaires, parfois beaucoup plus densément peuplés que celui que j'ai visité : survolant le territoire des Ande (un groupe de locuteur baruya situé immédiatement à l'ouest de Marawaka, au sud-ouest de la vallée), j'ai ainsi photographié des bosquets de Pandanus d'environ trois ou quatre hectares où s'élevaient une dizaine de maisons. Chacun des quinze clans baruya possède un tel endroit où dominent les Pandanus - entre 5 et 10 pour $100 \mathrm{~m}^{2}-$, quelque part dans la portion de forêt dont il a le monopole d'usage, si bien que c'est probablement une cinquantaine de maisons qui reçoivent chaque année pendant deux mois la visite de plusieurs centaines de personnes, résidant là à plein temps avec leurs cochons domestiques, leurs chiens et leurs enfants. Bref, pendant un sixième de l'année, une part non négligeable de

4. Mais il y a des Pandanus conoideus dans la partie la moins élevée des vallées occupées par les Wantekia (un groupe de locuteurs baruya, immédiatement au nord-ouest de Wonenara).

5. P. jiulianettii and P. brosimos sont très similaires (Barrau, $1962: 192-193$; Hyndman 1984, Sillitoe $1983: 103$; Stone 1992 : 241-242). Je me fie entièrement à Mike Bourke (comm. pers) qui m'a confirmé avoir observé les deux arbres à Marawaka (Bourke et al, 1994). Suivant Stone (1984 : 305), on peut sans doute parler ici d'un « brosimos-jiulianettii complex ». Je remercie Manuel Boissière et Claire Julliand pour l'abondante bibliographie qu'ils m'ont fournie.

6. Une semblable migration temporaire vers des bosquets de Pandanus est mentionnée par Schweinfurth (1984: 82). Barth (1975 : 41) signale que les Baktaman possèdent individuellement des bosquets individuels de Pandanus « à noix », mais je n'ai pas mention d'éventuels campements. Chez les Wopkaimin, des drupes de Pandanus brosimos sauvages sont collectées à proximité d'abris temporaires, mais il n'est fait mention d'aucun séchage Hyndman (1984 : 296). 
cette population d'horticulteurs éleveurs de porcs s'enfonce dans la forêt, bien au-dessus des jardins les plus élevés, pour collecter, surveiller et conserver les fruits d'un arbre «semidomestiqué » de la forêt.

Les Baruya disent que les drupes peuvent être stockées pendant près d'un an, mais le gros de la consommation des (délicieuses) amandes intervient clairement pendant la mauvaise saison (froide et très pluvieuse), entre novembre et janvier. Les amandes de Pandanus sont alors une friandise que l'on offre ou réclame plusieurs fois par jour à autrui. Comme le rappelait Jacques Barrau (1962 : 193) « [elles] sont riches en huile au point que des missionnaires catholiques français qu' [il a] visités dans les montagnes papoues s'en servent pour extraire leur huile de table et de friture ». Avec $66 \mathrm{~g}$ de graisse pour $100 \mathrm{~g}$ de matière sèche, les amandes sont, de loin, le plus riche des aliments que consomment les Baruya. C'est aussi celui qui contient le plus de protéines : $11,9 \mathrm{~g}$ pour $100 \mathrm{~g}$ (Powell, 1976) ${ }^{7}$.

À l'instar d'autres peuples de NouvelleGuinée, les Baruya consomment irrégulièrement des aliments qui, pour être « de complément», n'en tiennent pas moins une place essentielle dans leur diète - porc, marsupiaux, rats, anguilles, oiseaux, champignons, etc. Mais, à la différence des forêts de nombre de leurs voisins anga, celles des Baruya ne renferment pas d'arbres comme le Pandanus conoideus, le Pangium edule ou l'arbre à pain (Artocarpus camansi) dont la fructification saisonnière décalée leur permettrait d'avoir toute l'année accès à l'un ou l'autre de ces précieux « compléments ». Ils n'ont que les Pandanus « à noix », qui fructifient à la fin de l'année.

Comme l'a montré en 1997-1998 la sécheresse résultant d'un épisode El Niño particulièrement sévère, c'est également en fin d'année que la famine se fait sentir lorsque les pluies ne reviennent pas à partir de juin-juillet (Lemonnier, 2001). Avant l'arrivée de la patate douce, c'était aussi le moment où les taros Colocasia - qui constituaient alors l'essentiel de la nourriture des Baruya - pouvaient venir à manquer à la suite d'une sécheresse. Bien que rarissimes, ces épisodes de disette n'étaient pas inconnus. En tout cas, ce n'est pas forcer la réalité que d'avancer l'hypothèse que les Pandanus jiulianettii et $P$. brosimos - moins touchés par l'absence de pluie dans l'écrin humide que leur offre la forêt tropicale d'altitude -, offraient alors aux Baruya une nourriture de famine d'une exceptionnelle qualité. Au point que, longtemps après leur « sélection empirique » par l'« homme-cueilleur », les Pandanus continuent d'illustrer chez cette population installée depuis des millénaires dans les hautes terres de l'île l'un de ces " peuplements aménagés... dans le but d'en faciliter le ramassage » dont parlait J. Barrau (1967 : 281-282).

Comme tant de techniques, l'exploitation des $P$. jiulianettii et $P$. brosimos résulte d'un choix (Lemonnier, 1993) et tous les Pandanus des Anga ne sont pas également "semi-domestiqués». Les Ankave, par exemple, se contentent de brefs séjours - une nuit ou deux - dans les bosquets d'altitude, le temps de ramasser les syncarpes tombés des arbres ${ }^{8}$. Il est vrai que les Iqwaye qui, naguère, étaient réfugiés dans le haut de la vallée pourraient avoir l'idée d'en revendiquer indûment la propriété. Les Baruya, à l'inverse, ont fait le choix de ne rien partager avec les porcs sauvages. Du même coup, ces postes avancés de la culture des plantes que sont les bosquets yashwanala sont aussi les seuls lieux de production agricole qui, pendant plusieurs mois, demandent une présence de l'homme de tous les instants.

Une impression ne vaut que ce qu'elle vaut, mais l'atmosphère qui règne pendant quelques semaines sous les yota des Baruya n'est pas celle des hameaux où l'on réside le reste de l'année. Tout là-haut, dans la forêt d'altitude, au beau milieu des immenses territoires de chasse, c'est presque par famille que l'on se rassemble, par exemple autour de frères qui, seuls, peuvent cueillir les syncarpes de leurs Pandanus communs sans risquer de déclencher une vendetta. Chaque lignage veille sur ses arbres en même temps qu'il rappelle ses droits aux autres, avec l'aide des esprits des morts glajo qui gardent la forêt de chaque clan et tuent " tous ceux qu'ils voient pour la première fois "), c'est-à-dire ceux qui n'appartiennent ni au clan ni au lignage qui possède le droit d'usage de la portion de forêt en question (Kumain Nunguye, comm. pers.).

Là réside peut-être une partie des facteurs qui font des Pandanus des arbres pas comme les autres pour les Baruya (ou leurs proches cousins Sambia) : des arbres dont la sève peut secrètement remplacer le sperme perdu lors des rapports sexuels ou des boy-inseminating practices (Herdt, 1981 : 111) ; ou qui servent de théâtres végétaux lors des cérémonies d'initiation du premier stade (Dunlop, 1992: 28); des arbres auprès desquels la société baruya se morcelle par

7. Norgan et al (1979: 29) indiquent respectivement $43.8 \mathrm{~g}$ (graisse) and $11.7 \mathrm{~g}$ (protéines). La quantité de protéines et de graisses est trois fois plus élevée dans les amandes séchées que dans les amandes rôties (4.1 gr et $16.3 \mathrm{gr}$ ).

8. Le contraste entre les systèmes ankave et baruya est développé dans Bonnemère et Lemonnier (à paraître), publication qui reprend l'argument présenté ici. 
clans et lignages dont les membres résident et travaillent ensemble; des arbres, enfin, qui comptent au premier rang des marqueurs de territoire et pour lesquels on est prêt à se battre et mourir.

\section{BIBLIOGRAPHIE}

BARth, F., 1975. Ritual and Knowledge among the Baktaman of New Guinea. Oslo, New Haven, Universitetforlaget, Yale University Press.

BARRAU, J., 1962. Les plantes alimentaires de l'Océanie. Origines, distribution et usages. Marseille: Annales du musée colonial de Marseille.

—, 1965. Histoire et préhistoire horticoles de l'Océanie tropicale, Journal de la Société des Océanistes 21, pp. 55-78.

—, 1967. De l'homme cueilleur à l'homme cultivateur : l'exemple océanien, Cahiers d'Histoire Mondiale, pp. 275-292.

Bonnemere, P. et P. Lemonnnier, à paraître. Two Angan Vegeculture Systems in New Guinea. Technical ans Cultural Specificities in the Utilization of Some Seasonal Trees, in S. Yoshida, Y. Ochai, P. Matthews Vegeculture in Eastern Asia and Oceania, JCAS Symposium Series 16.

Dunlop ; I. Baruya Muka Archival, volume 5. Lindfield, Film Australia.

FeIL, D.K., 1987. The Evolution of Highlands Papua New Guinea Societies. Cambridge, Cambridge University Press.

Greve, J. van S., A.D. Hockung and A.K. Sharp, 1994. Traditional storage of pandanus nuts in the Papua New Guinea highlands, in Proceedings of the $6^{\text {th }}$ International Working Conference on Storedproduct Protection - Volume 2, pp. 1068-1074.

Herdt, G., 1981. Guardians of the Flutes. Idioms of Masculinity. New York, McGraw-Hill.

Hyndman, D.C., 1984. Ethnobotany of Wopkaimin Pandanus: Significant Papua New Guinea Plant Resource, Ethnobotany, 38(3), pp. 287-303.

KeLLy, R.C., 1988. Etoro suidology: areassessment of the pig's role in prehistory and comparative ethno- logy of New Guinea, in J.F. Weiner (ed), Mountain Papuans. Historical and comparative perspective from New Guinea Fringe Highlands societies, Ann Arbor, University of Michigan Press, pp. 111-186.

LEMONNIER, P., 1982. Jardins anga, Journal d'agriculture traditionnelle et de botanique appliquée, XIX (3-4), pp. 224-245.

—, 1984. La production de sel végétal chez les Anga (Papouasie Nouvelle-Guinée), Journal d'Agriculture Traditionnelle et de Botanique Appliquée, XXXI (1-2), pp. 71-126.

- 1990. Guerres et festins. Paix, échanges et compétition dans les Highlands de Nouvelle-Guinée. Paris, Éditions de la Maison des Sciences de l'Homme.

—, (éd.), 1993b. Technological Choices. Transformation in Material Cultures since the Neolithic. London: Routledge.

—, 2001. Drought, Famine and Epidemic Among the Ankave-Anga of Gulf Province in 1997-98, in R.M. Bourke, M.G. Allen and J.G. Salisbury (eds), Food Security for Papua New Guinea. Canberra, Australian Center for International Agricultural Research (ACIAR Proceedings n ${ }^{\circ}$ 99), pp. 164-167.

Lloyd, J.A., 1992. A Baruya - Tok Pisin - English Dictionary. Canberra, Pacific Linguistics (Series C-no 82$)$.

LORY, J.-L., 1982. Les Jardins baruya, Journal d'Agriculture Traditionnelle et de Botanique Appliquée, 29 (3-4), pp. 247-274.

Norgan, N.G., J.V.G.A., Durnin and A. FerroLuzzI, 1979. The composition of some New Guinea foods, Papua New Guinea Agriculture Journal 30(1), pp. 25-39.

Powell, J.M., 1976. Ethnobotany, in K. Paijmans (ed), New Guinea Vegetation. Canberra, CSIRO, pp. 106-183.

SChweInfurth, U., 1984. Man and Environment in the Central Cordillera of Eastern New Guinea. Pandanus, Casuarina, Ipomoea batatas, Erdwissenschaftliche Forschung 18, pp. : 79-98.

Sillitoe, P., 1983. Roots of the earth. Crops in the Highlands of Papua New Guinea. Kensington (Australia), New South Wales University Press.

Stone, B.C., 1992. Pandanus Parkinson, Prosea 2, pp. 240-243. 